\title{
Limited brain metastases: a narrative review
}

\author{
Kevin Victor Chaung ${ }^{1}$, Andrew Edward Sloan ${ }^{2,3}$, Serah Choi ${ }^{1,3}$ \\ ${ }^{1}$ Department of Radiation Oncology, University Hospitals Cleveland Medical Center, Cleveland, OH, USA; ${ }^{2}$ Department of Neurological Surgery, \\ University Hospitals Cleveland Medical Center, Cleveland, OH, USA; ${ }^{3}$ Case Western Reserve University School of Medicine, Cleveland, OH, USA \\ Contributions: (I) Conception and design: KV Chaung, S Choi; (II) Administrative support: None; (III) Provision of study materials or patients: None; \\ (IV) Collection and assembly of data: KV Chaung, S Choi; (V) Data analysis and interpretation: All authors; (VI) Manuscript writing: All authors; (VII) \\ Final approval of manuscript: All authors. \\ Correspondence to: Serah Choi, MD, PhD. 11100 Euclid Ave, Lerner Tower B163, Cleveland, OH 44106, USA. Email: serah.choi@uhhospitals.org.
}

\begin{abstract}
Limited brain metastases refer to an oligometastatic state in the brain for which focal therapy with stereotactic radiosurgery (SRS) is appropriate. The definition of what is considered "limited" for brain metastases, however, is not well defined. Multiple recent randomized trials show that metastasis-directed therapy with surgery or stereotactic radiation can prolong survival for patients with 1-5 metastases from various primary tumors, but patients with untreated brain metastases were largely excluded from these trials. Stereotactic radiosurgery allows for the treatment of multiple brain metastases while avoiding whole brain radiation (WBRT), which has a known negative impact on neurocognitive function. Randomized trials have shown that stereotactic radiation alone is effective for the treatment of up to 4 brain metastases with decreased neurocognitive side effects and no detriment to overall survival (OS). These data have led to our current definition of "limited" brain metastases. However, more recent data suggests that patients with up to 10 brain metastases can benefit from SRS. Ongoing trials are investigating if patients with up to 15-20 brain metastases may similarly benefit from SRS alone. Advances in systemic therapies which penetrate the bloodbrain barrier and have greater activity in central nervous system (CNS) may also expand the population for whom radiosurgery is an appropriate treatment. Immunotherapy may also be synergistic with radiosurgery in some cases. This narrative review will discuss the evolving definition and management of limited brain metastases.
\end{abstract}

Keywords: Brain metastases; oligometastatic disease; stereotactic radiosurgery (SRS); targeted therapy; immunotherapy

Submitted Feb 09, 2021. Accepted for publication Mar 23, 2021.

doi: 10.21037/apm-21-363

View this article at: http://dx.doi.org/10.21037/apm-21-363

\section{Introduction}

Historically, cancer patients with any distant metastatic disease were considered incurable, with little chance of long-term survival. The prognosis for brain metastases was particularly poor, and the primary treatment was palliative whole brain radiation (WBRT). However, more recent studies suggest that patients with metastatic disease often present with a limited number of metastatic sites that are amenable to local therapy (1-3). "Oligometastatic disease" refers to an intermediate clinical state in a spectrum between localized disease and diffuse metastatic spread. In this model, a patient with only a few distant metastases may have a tumor biology with limited potential for metastatic spread compared to a patient with more extensive disease (4). These oligometastatic patients thus may be treated with curative intent with aggressive metastasis-directed local therapy using surgery or ablative doses of radiation. We use the term "limited brain metastases" to refer to an oligometastatic state in the brain that is appropriate to treat with more aggressive local ablative therapies to achieve a longer overall survival (OS) or spare neurocognitive function. The definition of "limited" in terms of number or volume of lesions is fluid and evolving; it has been defined 
in multiple randomized clinical trials as up to 4 brain metastases, but recent data suggests that up to 10 brain metastases may be appropriate or that the "limit" may be better defined by the cumulative intracranial tumor volume. The introduction of novel systemic therapies with improved intracranial activity compared to traditional cytotoxic chemotherapy may also expand our definition of limited brain metastases. The purpose of this review is to discuss the evolution of the definition and management of limited brain metastases in the context of oligometastatic disease. We present the following article in accordance with the Narrative Review reporting checklist (available at http:// dx.doi.org/10.21037/apm-21-363).

\section{Methods}

The PubMed database was searched using the terms "oligometastatic disease", "brain metastases", "stereotactic radiosurgery", "targeted therapy", and "immunotherapy" for articles published in the English language from 1960 through January 2021. Data from review articles, randomized controlled trials, non-randomized trials, retrospective studies, and conference abstracts were included in this narrative review.

\section{Discussion}

\section{Oligometastatic disease and brain metastases}

Our understanding of the biological mechanisms underlying metastatic spread remains incomplete, but significant progress has been made in identifying a multistep sequence of events known as the invasion-metastasis cascade. Cells from the primary tumor must gain the ability for local invasion, followed by loss of intercellular adhesion and spread into the circulatory system. These circulating tumor cells (CTCs) can then extravasate into distant organs to form micrometastatic colonies. The final stage involves proliferation of these colonies in distant organs, each with a distinct microenvironment to which the cancer cells must adapt if they are to survive and form clinically detectable lesions (5-7). In the case of brain metastases, cancer cells must cross the blood-brain barrier, which is composed of endothelial cells lined by basement membrane, pericytes, and astrocyte foot processes. They then must evade astrocyte-induced apoptosis and other features of the microenvironment that suppress tumor proliferation (7). There is some evidence that different primary tumor types have different tropism for the brain (8). Genomic profiling studies are just beginning to elucidate features of brain metastasis cells that distinguish them from the primary tumor or other extracranial metastases (9). Heterogeneity in the ability of cancer cells to complete the different steps of the invasionmetastasis cascade may account for the observed spectrum of oligometastatic and polymetastatic disease. One patient's cancer may be particularly adept at leaving the primary tumor and entering circulation but not able to overcome the microenvironment of a distant organ, while another patient may have a low number of CTCs that nonetheless are able to efficiently colonize distant organs (10). Currently, there are no validated biomarkers for oligometastatic disease, and further understanding of what affects a cancer cell's potential for metastatic spread will be necessary to refine our current definitions $(11,12)$.

There remains controversy over how to define oligometastatic disease in terms of the maximum number of metastatic lesions, their maximum size, their distribution among different organs, and if these factors vary depending on the primary tumor histology. The European Society for Radiotherapy and Oncology (ESTRO) and the American Society for Radiation Oncology (ASTRO) recently published a consensus statement defining oligometastatic disease as $1-5$ metastatic lesions that can be safely treated with local therapy, regardless of the primary histology, the metastatic site, or the time between diagnosis of the primary tumor and appearance of metastases. Of note, the authors also recommended that patients with intracranial metastases should not be excluded from trials of oligometastatic patients (11).

There is increasing evidence that metastasis-directed therapy can improve disease control and ultimately survival in the oligometastatic population. Early data came from retrospective series of oligometastatic patients who underwent resection of lung or liver metastases, some of whom achieved long-term disease control and survival (13-16). Advances in technology introduced stereotactic body radiation therapy (SBRT), a method of delivering ablative doses of radiation to focal sites of disease while sparing nearby local tissue, as an alternative to surgery for local control of extracranial metastatic sites (17-20). Multiple randomized trials have also demonstrated the survival benefit of metastasis-directed therapy in patients with 1-5 metastases of varying primary histologies (21-28).

Although the ESTRO-ASTRO consensus statement did not distinguish extracranial from intracranial metastases in 
its definition of oligometastatic disease (11), most of these randomized trials of metastasis-directed therapy specifically excluded brain metastases. For example, the SABRCOMET trial did allow patients with brain metastases, but excluded those with 1-3 brain metastases without any other sites of disease, as these patients would not be recommended to forego local therapy. Consequently, only four out of a total of 191 metastatic lesions in this trial were brain metastases (21).

Up to $30 \%$ of cancer patients develop brain metastases during their disease course, most frequently from lung cancer, breast cancer, renal cell carcinoma (RCC), and melanoma $(8,29-32)$. The incidence has increased with the advent of magnetic resonance imaging (MRI), which quickly replaced computed tomography (CT) as the imaging modality of choice for intracranial lesions. Thin-slice MRIs and volumetric image acquisition have also further enhanced our ability to detect much smaller lesions (33). Brain metastases represent a special case in the oligometastatic paradigm. Obtaining upfront local control of intracranial metastases with surgery and/or radiation is typically standard of care, as the blood-brain barrier prevents many systemic therapies from controlling disease in the central nervous system (CNS), and brain metastases can rapidly progress without local treatment (34). Thus, patients with untreated brain metastases have largely been excluded from randomized trials examining the benefit of metastasis-directed therapy in oligometastatic patients, including the ongoing national randomized phase II/III trials NRG-LU002 and NRG-BR002. Prognosis after development of brain metastases has historically been poor overall, but a subset of brain metastasis patients may live for years after diagnosis, especially those with "limited" intracranial disease or targetable molecular alterations (9).

Defining an oligometastatic state for brain metastases is challenging. Multiple factors, including the number of detectable brain lesions, have been found to contribute to prognosis. Multiple prognostic indices and nomograms have been devised over the past few decades to help identify longer-lived patients (35-39). Gaspar et al. developed a recursive partitioning analysis (RPA) of patients from three consecutive RTOG brain metastasis studies to separate patients into three classes with differing survival based on Karnofsky Performance Status (KPS), age, control of the primary tumor, and presence of extracranial metastases (35). However, the RPA classification did not take into account the number of brain metastases and relied on a subjective estimation of primary tumor control. Sperduto et al. thus introduced a Graded Prognostic Assessment (GPA) based on analysis of five randomized RTOG brain metastasis studies. The GPA incorporated age, KPS, number of brain metastases, and presence of extracranial metastases, and weighted each of these factors based on their prognostic significance (36). As the significance of primary tumor histology on brain metastasis outcomes became more apparent, the GPA was refined into a diagnosis-specific GPA (DS-GPA) using a separate multi-institutional database of patients with brain metastases from breast cancer, non-small cell lung cancer (NSCLC), small cell lung cancer (SCLC), renal cell carcinoma (RCC), melanoma, and gastrointestinal cancers. Significant prognostic factors varied by primary tumor histology; for example, the DS-GPA breast cancer includes tumor subtype (luminal A, luminal B, HER2overexpressing, or basal) (37). Further updates to the DSGPA have also incorporated clinically actionable molecular features, such as EGFR mutation and ALK rearrangement for NSCLC. While the most recent DS-GPAs all include the number of brain metastases as a prognostic factor, the numbers used to stratify outcomes differ by primary histology. For example, the breast DS-GPA separates patients with a single brain metastasis from those with two or more, while the NSCLC DS-GPA separates them into those with 1-4 metastases versus those with 5 or more, suggesting that influence of oligometastatic brain metastases may vary by histology. Absence of extracranial metastases has consistently remained a strong positive prognostic factor across all the DS-GPAs, consistent with our understanding of oligometastatic disease (38). As systemic treatment options have improved, survival for the most favorable patients has greatly improved. Patients with the best prognosis under the RPA classification had a median OS of 7.1 months, but the patients with the best prognosis under the most recent DS-GPA for NSCLC (score 3.5-4.0) had a median OS of up to 46 months.

\section{Surgical resection for single brain metastasis}

Perhaps the best evidence we have for aggressive metastasisdirected therapy in the brain improving OS is in the setting of a single brain metastasis. Historically, the standard treatment for brain metastases was palliative conventionally fractionated WBRT alone. However, retrospective and nonrandomized studies showed a survival benefit from surgical resection for a single brain metastasis. Patchell et al. performed a prospective trial randomizing 48 patients with a single brain metastasis to biopsy followed by WBRT 
versus surgical resection followed by WBRT. The addition of surgery to WBRT had significantly improved median OS (40 vs. 15 weeks, $\mathrm{P}<0.01)$ and remained functionally independent for significantly longer (38 vs. 8 weeks, $\mathrm{P}<0.005)$ (40). Similarly, Noordijk et al. showed in a prospective randomized trial of 63 patients with a single brain metastasis that surgery plus WBRT significantly improved median survival compared to WBRT alone (10 vs. 6 months, $\mathrm{P}=0.04$ ). Notably, the patients who had active extracranial disease had a median survival of only 5 months regardless of the treatment arm, and patients older than 60 years had significantly higher mortality compared to younger patients (HR 2.74, $\mathrm{P}=0.003$ ) (41). Another trial by Patchell et al. looked at the role of WBRT after surgical resection of a single brain metastasis. In this study, 95 patients with one brain metastasis and KPS $\geq 70$ were randomized between surgery alone versus surgery followed by WBRT. The postop WBRT arm had decreased intracranial failure rates $(18 \%$ vs. $70 \%, \mathrm{P}<0.001)$ and local recurrence $(10 \%$ vs. $46 \%, \mathrm{P}<0.001)$, but no significant difference in median OS ( 48 vs. 43 weeks, $\mathrm{P}=0.39$ ). It should be noted that this study was powered to detect differences in local control and not survival, however (42). Taken together, these trials suggest that aggressive local control of a single brain metastasis with surgical resection can improve OS for selected patients, analogous to the results of the recent randomized trials of metastasis-directed therapy for 1-5 extracranial metastases.

\section{What about local therapy for more than a single brain metastasis?}

Although WBRT offers effective intracranial local and distant control for multiple brain metastases, the resulting neurocognitive function impairment makes it a less desirable first-line option when focusing on quality of life. Technological advancements have enabled the treatment of multiple brain metastases with stereotactic radiosurgery (SRS). SRS was developed in the 1950s by Lars Leksell as a noninvasive method of treating small intracranial targets by delivering focal high dose radiation (often in a single fraction) with sharp dose fall-off, thereby sparing normal brain tissue, analogous to the use of SBRT for extracranial tumors (43). The first SRS treatments were done using Gamma Knife radiosurgery (GKRS), but linear acceleratorbased SRS has also been available since the 1980s (44). GKRS was originally developed for functional neurosurgery, but it was quickly accepted as a treatment for benign intracranial tumors and arteriovenous malformations, and eventually brain metastases (43-45).

Over the past 10-15 years, SRS alone has become the standard of care for patients with limited brain metastases, which the National Comprehensive Cancer Network (NCCN) currently defines as "patients for whom SRS represents an effective alternative to WBRT, but with more cognitive protection." However, the NCCN definition of "limited" in terms of number or volume of lesions is not strictly defined (46). Although SRS has not been shown to improve survival compared to WBRT for more than one brain metastasis, the goal of ablative radiation is not necessarily for curative treatment. Brain metastasis patients often have active extracranial disease, especially if they present with synchronous brain metastases. Instead, one of the primary goals of SRS is local control of intracranial disease while limiting radiation dose to the normal brain tissue as much as possible. Although omitting upfront WBRT may lead to increased rates of intracranial progression by not treating potential micrometastatic disease not detected on imaging, multiple randomized trials have shown improved neurocognitive function and quality of life with SRS alone compared to SRS plus WBRT, without significant decrease in OS.

RTOG 9508 was a multi-institutional trial of 333 patients with 1-3 newly diagnosed brain metastases who were randomized to WBRT versus WBRT followed by SRS boost. Patients in the SRS boost arm had significantly improved local control at 1 year $(82 \%$ vs. $71 \%, \mathrm{P}=0.013)$ but did not have improved median OS $(5.7$ vs. 6.5 months, $\mathrm{P}=0.136)$. However, patients with a single brain metastasis did have improved median OS with SRS (6.5 vs. 4.9 months, $\mathrm{P}=0.039)$, similar to the findings of Patchell et al. and Noordijk et al. (47).

The JROSG 99-1 trial was a multi-institutional trial of 132 patients with $1-4$ brain metastases each $\leq 3 \mathrm{~cm}$ in greatest diameter ( $49 \%$ had a single brain metastasis) who were randomly assigned to WBRT plus SRS versus SRS alone. Median OS (the primary endpoint) was not significantly different between the two arms (7.5 vs. 8.0 months, $\mathrm{P}=0.42$ ), but the SRS alone arm had a higher rate of 12 -month total intracranial recurrence $(76.4 \%$ vs. $46.8 \%, \mathrm{P}<0.001)$, local recurrence $(27.5 \%$ vs. $11 \%$, $\mathrm{P}=0.002)$, and distant recurrence $(64 \%$ vs. $42 \%, \mathrm{P}=0.003)$. The study was ended early after interim analysis showed futility in detecting a significant difference in OS (48). A secondary analysis of this trial stratified the subset of 88 NSCLC patients by DS-GPA and found that the subset of 
47 patients with NSCLC and favorable prognosis with GPA scores between 2.5 and 4.0 had improved median survival with the addition of WBRT (16.7 vs. 10.6 months, $\mathrm{P}=0.04$ ), suggesting that perhaps more aggressive intracranial control with WBRT plus SRS might be beneficial for longer surviving patients (49).

A phase III study from MD Anderson evaluated patients with 1-3 brain metastases and randomized them to SRS versus SRS plus WBRT. The primary endpoint was decline in neurocognitive function defined as a significant deterioration (5-point drop from baseline) in the Hopkins Verbal Learning Test-Revised (HVLT-R) at 4 months. A total of 58 patients were enrolled, and the trial was ended early after interim analysis showed a $96 \%$ probability that the SRS plus WBRT arm would be significantly more likely to have a decline in neurocognitive function. Similar to the JROSG-99-1 trial, the SRS alone arm did have a higher rate of $\mathrm{CNS}$ recurrence at 1 year $(73 \%$ vs. $27 \%, \mathrm{P}=0.0003)$. Surprisingly, median OS was significantly higher in the SRS alone arm (15.2 vs. 5.7 months, $\mathrm{P}=0.003)$, which the authors speculated could be related to more surgical salvage, earlier systemic therapy, or lower burden of extracranial disease in the SRS alone arm (50).

EORTC 22952 was a phase III trial of patients with 1-3 brain metastases and WHO performance status (PS) 0-2 who underwent SRS or surgery, and then were randomized to WBRT or observation. The primary endpoint was time to WHO PS deterioration to greater than 2, which was not significantly different between WBRT and observation (9.5 vs. 10.0 months, $\mathrm{P}=0.71$ ) even though there was a higher rate of intracranial recurrence in the observation arm. Median OS was also similar between the arms (10.9 vs. 10.7 months, $\mathrm{P}=0.89$ ) (51). A follow-up publication reported health-related quality-of-life (HRQOL) results, which was a secondary endpoint. Compliance with HRQOL assessments dropped to only $45 \%$ at 1 year, and so only the first year was analyzed. Patients in the WBRT arm had significantly worse HRQOL scores for global health status at 9 months, physical functioning at 8 weeks, cognitive functioning at 12 months, and fatigue at 8 weeks (52).

An individual patient data meta-analysis of the above three studies (JROSG 99-1, the MD Anderson trial, and EORTC 22952) compared patients who received SRS alone versus SRS plus WBRT. Patient's age 50 or younger had significantly better median OS with SRS alone (10 vs. 8.2 months, $\mathrm{P}=0.04$ ) without a significant difference in distant brain failure. Patients with a single brain metastasis also had better median OS and lower risk of distant brain failure compared to those with 2-4 metastases (53).

The North Central Cancer Treatment Group (NCCTG) N0574 phase III trial randomized 208 patients with 1-3 brain metastases between SRS alone versus SRS plus WBRT. The primary endpoint was cognitive deterioration defined as greater than one standard deviation from baseline on at least one of seven cognitive tests at three months postradiation treatment. At three months, the SRS group had a significantly lower rate of cognitive deterioration compared to the SRS plus WBRT group (63.5\% vs. 91.7\%, $\mathrm{P}<0.001)$. For long term survivors (patients who had a cognitive evaluation more than 12 months after randomization), the rate of cognitive deterioration was also less for the SRS alone arm at 12 months ( $60 \%$ vs. $94.4 \%, \mathrm{P}=0.04)$. The SRS alone group did have a significantly shorter time to intracranial failure (HR 3.6, $\mathrm{P}<0.001$ ), but there was no significant difference in median OS between the two arms (10.4 months for SRS alone vs. 7.4 months for SRS plus WBRT, $\mathrm{P}=0.92$ ) (54).

Another multi-institutional phase III trial led by Melanoma Institute Australia included patients with 1-3 melanoma brain metastases who had local treatment with SRS or surgery, and randomized them to WBRT versus observation. The primary endpoint was distant intracranial failure within 12 months, which was not significantly different between the two arms (42\% for WBRT vs. $50.5 \%$ for observation, $\mathrm{P}=0.22)$. The local failure rate was lower for the WBRT group (20.0\% vs. 33.6\%, $\mathrm{P}=0.03$ ), but there was no significant difference in 12 -month OS $(58.5 \%$ for WBRT vs. $48.6 \%$ for observation, $\mathrm{P}=0.28$ ) or median time to deterioration in performance status (3.8 months for WBRT vs. 4.4 months for observation, $\mathrm{P}=0.32$ ) (55).

Most of the aforementioned trials comparing SRS with or without WBRT excluded SCLC because of the high incidence of brain metastases and historically poor outcomes, but recent retrospective data show that upfront SRS may also be an appropriate treatment option for SCLC patients with limited brain metastases. The FIRE-SCLC multicenter non-randomized cohort study included 710 SCLC brain metastasis patients who received upfront SRS without prior prophylactic cranial irradiation or WBRT, and compared them to a separate WBRT cohort. Time to CNS progression was shorter for the SRS group, but without a detriment in median OS (6.5 months for upfront SRS vs. 5.2 months for WBRT, $\mathrm{P}=0.003$ ) (56).

In summary, the development of SRS has allowed for focal treatment of up to 4 brain metastases, with decreased neurocognitive side effects and similar OS when compared 
to WBRT. SRS alone has thus become the standard upfront therapy for patients with 1-4 brain metastases. Accordingly, the ASTRO Choosing Wisely campaign recommended in 2014 that WBRT should not be routinely added to SRS for limited brain metastases (57).

\section{What about more than 4 brain metastases?}

Early SRS treatments were typically limited to $1-3$ brain lesions owing to practical concerns. Until the mid-2000s, GKRS for multiple lesions required helmet changes and manual plugging of collimators, and linear acceleratorbased SRS required cone-based collimation with specific quality assurance for each target lesion. Advancements in technology have allowed for treatment of greater numbers of lesions in each patient. Multiple retrospective series on patients treated with GKRS alone for greater than $8-10$ brain metastases suggested that this was feasible without compromising local control or survival (58-62). In 2014, Yamamoto et al. reported results from a Japanese multicenter prospective trial (JLGK0901) of 1,194 patients with 1-10 brain metastases treated with GKRS alone. The cumulative tumor volume was limited to $\leq 15 \mathrm{cc}$, with the largest tumor $<10 \mathrm{cc}$. The patients were grouped by number of metastases: one lesion, 2-4 lesions, and 5-10 lesions. Between patients with 2-4 lesions versus 5-10 lesions, there was no significant difference in median OS (10.8 months for both groups, HR $0.97, \mathrm{P}=0.78$ ) or rates of treatment-related adverse events ( $9 \%$ for both groups, $\mathrm{P}=0.89$ ). Across all three groups, there was no difference in the rate of appearance of new lesions, local recurrence, leptomeningeal dissemination, or use of salvage SRS or WBRT. Patients with a single brain lesion had a median OS of 13.9 months, which was significantly improved compared to those with 2-4 lesions ( $\mathrm{P}=0.0001)$ (63). Maintenance of neurocognitive function (defined as those patients whose Mini-Mental Status Exam score did not decrease by $\geq 3$ points from baseline) was also similar regardless of the number of lesions, though this test is less sensitive for detecting changes in function compared to other validated cognitive tests such as the HVLT-R, the Controlled Oral Word Association (COWA) test, or the Trail Making Test (TMT) $(64,65)$.

More recently, preliminary results from a phase III randomized controlled trial of patients with 4-15 nonmelanoma brain metastases were presented at the ASTRO 2020 Annual Meeting in abstract form. Seventy-two patients with a median of 8 brain metastases at enrollment were randomly assigned to upfront SRS or WBRT. Neurocognitive function testing was done at baseline and longitudinally. Local control at 4 months was $100 \%$ for the SRS group and $95.5 \%$ for the WBRT group $(\mathrm{P}=0.53)$, and median OS was not significantly different $(\mathrm{P}=0.45)$. Neurocognitive function at 4 months improved on average in the SRS arm while it declined in the WBRT arm $(\mathrm{P}=0.008)$ (66).

SRS has been used to treat even greater numbers of brain metastases, but as the number of detectable metastases increases, so does the risk of distant intracranial failure if WBRT is omitted $(67,68)$. The amount of integral radiation dose going to the normal brain tissue also increases with a greater number of treated lesions, which could diminish the relative neurocognitive benefit of omitting WBRT in these patients. Multiple ongoing prospective trials will examine whether SRS alone can preserve neurocognitive function without compromising local control or survival compared to WBRT for patients with up to 15-20 brain lesions (NCT03550391, NCT01592968, NCT03075072, NCT03775330). These trials notably exclude SCLC and other radiosensitive primary histologies, but the upcoming phase III NRG-CC009 trial will randomize SCLC brain metastasis patients between upfront SRS and hippocampalavoidant WBRT for 10 or fewer brain lesions (69).

\section{What about the volume of brain metastases?}

In addition to the number of brain lesions, the size and volume of the lesions also affect the feasibility of SRS. For example, a patient may have a single brain metastasis that is too large to be effectively treated; as the size of the lesion grows, the dose for single fraction SRS must be lowered accordingly to avoid excess risk of radiation necrosis (70). As the dose decreases, local control also decreases as a result; local control at 12 months can fall below $50 \%$ with a dose of $\leq 15$ Gy to the tumor margin, compared to $>80 \%$ with a dose of $\geq 20$ Gy $(71,72)$. For larger tumors, fractionated SRS (typically 3-5 fractions) has been used to improve local control rates while maintaining sharp dose gradients that spare normal brain tissue, with radiation necrosis rates comparable to those seen with single fraction SRS to smaller lesions $(73,74)$. Although fractionated SRS allows patients to avoid WBRT for larger tumors, cumulative intracranial tumor volume may represent a prognostic factor in addition to the number of brain metastases. Multiple retrospective series of brain metastasis patients have found increasing cumulative tumor volume to 
be associated with worse OS, local tumor progression, and distant brain failure (75-78). Conversely, the JLGK0901 trial, which compared outcomes of SRS for one, 2-4, and $5-10$ brain metastases and allowed for a cumulative brain metastases volume of up to $15 \mathrm{cc}$, did not find a significant association between OS and cumulative tumor volume on multivariable analysis (63).

\section{How will novel systemic therapies change our definition of limited brain metastases?}

The blood-brain barrier has limited the role of traditional cytotoxic chemotherapy in the treatment of intracranial disease. The discovery of targetable molecular alterations in subsets of cancer patients has led to the development of targeted therapies with better blood-brain barrier penetration and greater efficacy in the treatment of brain metastases, which is why these alterations are included in the most updated DS-GPAs (38). EGFR mutations are present in up to $35 \%$ of NSCLC cases, and the third-generation EGFR tyrosine kinase inhibitor (TKI) osimertinib has been shown to have favorable intracranial response rates $(79,80)$. Ongoing clinical trials are examining the use of osimertinib with and without SRS for asymptomatic brain metastases (NCT03535363, NCT03769103). Similarly, novel ALK inhibitors such as alectinib and brigatinib have also shown some efficacy for ALK-mutated NSCLC brain metastasis patients $(81,82)$. For HER2-positive breast cancer, the combination of lapatinib and capecitabine has shown CNS activity (83-85), as has the combination of neratinib and capecitabine (86) and the combination of tucatinib, capecitabine, and trastuzumab $(87,88)$. BRAF-mutated melanoma brain metastases have been successfully treated using BRAF inhibitors with or without MEK inhibitors $(89,90)$. The ongoing phase II Alliance A071701 trial (NCT03994796) is investigating the use of CDK, PI3K/ mTOR, and NTRK/ROS1 inhibitors for brain metastases harboring genomic alterations associated with sensitivity to these drugs (91).

The development of immune-checkpoint inhibitors (ICIs), including anti-PD-1, anti- PD-L1, and antiCTLA-4 antibodies, has also greatly changed the treatment paradigm for multiple types of cancer, and these agents have now become part of the standard of care for NSCLC, $\mathrm{RCC}$, and melanoma in particular. Immunotherapy alone has shown promise in treating asymptomatic brain metastases, especially for NSCLC and melanoma (92-94). Data from retrospective series suggest synergy between concurrent ICIs and SRS which can improve intracranial tumor response, response durability, and OS compared to SRS alone or sequential administration of ICIs and SRS. This is presumed to be due to enhanced systemic anti-tumor immune activity, known as the abscopal effect $(95,96)$. However, the potential benefits of concurrent immunotherapy and SRS must be balanced with potential toxicities, as the risk of radiation necrosis may increase with this combination based on retrospective data (97).

Targeted therapies and immunotherapy may become standard adjunct therapies to focal radiation for brain metastases in selected patients. If SRS treats macroscopic sites of disease, then these novel systemic therapies might be used to treat potential microscopic disease elsewhere in the brain and delay distant intracranial progression, thereby acting as an alternative to WBRT. A patient who initially presents with extensive brain metastases might also be converted to having limited disease after systemic treatment, thus becoming a candidate for SRS without WBRT.

\section{Summary}

Oligometastatic disease is a proposed clinical state marked by low metastatic burden and more indolent behavior compared to diffuse metastases. Our understanding of the biological underpinnings of the oligometastatic state remains limited, and there are currently no validated biomarkers. Related to oligometastasis is the concept of limited brain metastases, referring to a subset of brain metastasis patients with intracranial disease that can be reasonably treated using SRS and/or surgery while safely avoiding WBRT and its neurocognitive side effects. Our definition of limited brain metastases has been based on multiple randomized trials showing the efficacy of SRS alone for 1-4 brain metastases from a variety of primary histologies without compromising OS by omitting WBRT, but ongoing randomized trials may increase this number to 10 or greater, and emerging data suggests that total intracranial tumor volume is also an important factor. The introduction of targeted therapies and immunotherapies that offer improved blood-brain barrier penetration and intracranial activity compared to traditional cytotoxic chemotherapies have significantly improved survival outcomes for a subset of brain metastasis patients. These novel systemic agents are opening new avenues of management for brain metastases beyond local therapy, and may expand the patient population for whom SRS is appropriate and thus our definition of limited brain metastases. 


\section{Acknowledgments}

Funding: This work was supported by the National Institutes of Health (grant number K12CA076917 to S.C.); the Center of Excellence for Translational NeuroOncology, The Peter D. Cristal Chair, and the Kimble Family Fund (A.E.S.).

\section{Footnote}

Provenance and Peer Review: This article was commissioned by the Guest Editors (Simon Lo, Michael Milano, Tithi Biswas, Charles Simone) for the series "OligometastasisFallacy or Real Deal?" published in Annals of Palliative Medicine. The article has undergone external peer review.

Reporting Checklist: The authors have completed the Narrative Review reporting checklist. Available at http:// dx.doi.org/10.21037/apm-21-363

Peer Review File: Available at http://dx.doi.org/10.21037/ apm-21-363

Conflicts of Interest: All authors have completed the ICMJE uniform disclosure form (available at http://dx.doi. org/10.21037/apm-21-363). The series "OligometastasisFallacy or Real Deal?" was commissioned by the editorial office without any funding or sponsorship. SC received some salary support from the K12CA076917 grant (started January 2020). The authors have no other conflicts of interest to declare.

Ethical Statement: The authors are accountable for all aspects of the work in ensuring that questions related to the accuracy or integrity of any part of the work are appropriately investigated and resolved.

Open Access Statement: This is an Open Access article distributed in accordance with the Creative Commons Attribution-NonCommercial-NoDerivs 4.0 International License (CC BY-NC-ND 4.0), which permits the noncommercial replication and distribution of the article with the strict proviso that no changes or edits are made and the original work is properly cited (including links to both the formal publication through the relevant DOI and the license). See: https://creativecommons.org/licenses/by-nc-nd/4.0/.

\section{References}

1. Hong JC, Salama JK. The expanding role of stereotactic body radiation therapy in oligometastatic solid tumors: What do we know and where are we going? Cancer Treat Rev 2017;52:22-32.

2. Mehta N, Mauer AM, Hellman S, et al. Analysis of further disease progression in metastatic non-small cell lung cancer: implications for locoregional treatment. Int J Oncol 2004;25:1677-83.

3. Tosoian JJ, Gorin MA, Ross AE, et al. Oligometastatic prostate cancer: definitions, clinical outcomes, and treatment considerations. Nat Rev Urol 2017;14:15-25.

4. Hellman S, Weichselbaum RR. Oligometastases. J Clin Oncol 1995;13:8-10.

5. Massagué J, Obenauf AC. Metastatic colonization by circulating tumour cells. Nature 2016;529:298-306.

6. Lambert AW, Pattabiraman DR, Weinberg RA. Emerging Biological Principles of Metastasis. Cell 2017;168:670-91.

7. Obenauf AC, Massague J. Surviving at a Distance: OrganSpecific Metastasis. Trends Cancer 2015;1:76-91.

8. Barnholtz-Sloan JS, Sloan AE, Davis FG, et al. Incidence proportions of brain metastases in patients diagnosed (1973 to 2001) in the Metropolitan Detroit Cancer Surveillance System. J Clin Oncol 2004;22:2865-72.

9. Suh JH, Kotecha R, Chao ST, et al. Current approaches to the management of brain metastases. Nat Rev Clin Oncol 2020;17:279-99.

10. Reyes DK, Pienta KJ. The biology and treatment of oligometastatic cancer. Oncotarget 2015;6:8491-524.

11. Lievens Y, Guckenberger M, Gomez D, et al. Defining oligometastatic disease from a radiation oncology perspective: An ESTRO-ASTRO consensus document. Radiother Oncol 2020;148:157-66.

12. Pitroda SP, Weichselbaum RR. Integrated molecular and clinical staging defines the spectrum of metastatic cancer. Nat Rev Clin Oncol 2019;16:581-8.

13. Tomlinson JS, Jarnagin WR, DeMatteo RP, et al. Actual 10-year survival after resection of colorectal liver metastases defines cure. J Clin Oncol 2007;25:4575-80.

14. Wilkins EW Jr, Burke JF, Head JM. The surgical management of metastatic neoplasms in the lung. J Thorac Cardiovasc Surg 1961;42:298-309.

15. van Dongen JA, van Slooten EA. The surgical treatment of pulmonary metastases. Cancer Treat Rev 1978;5:29-48.

16. Fong Y, Fortner J, Sun RL, et al. Clinical score for 
predicting recurrence after hepatic resection for metastatic colorectal cancer: analysis of 1001 consecutive cases. Ann Surg 1999;230:309-18; discussion 18-21.

17. Pan H, Simpson DR, Mell LK, et al. A survey of stereotactic body radiotherapy use in the United States. Cancer 2011;117:4566-72.

18. Rusthoven KE, Kavanagh BD, Burri SH, et al. Multiinstitutional phase I/II trial of stereotactic body radiation therapy for lung metastases. J Clin Oncol 2009;27:1579-84.

19. Rusthoven KE, Kavanagh BD, Cardenes H, et al. Multiinstitutional phase I/II trial of stereotactic body radiation therapy for liver metastases. J Clin Oncol 2009;27:1572-8.

20. Milano MT, Katz AW, Zhang H, et al. Oligometastases treated with stereotactic body radiotherapy: long-term follow-up of prospective study. Int J Radiat Oncol Biol Phys 2012;83:878-86.

21. Palma DA, Olson R, Harrow S, et al. Stereotactic ablative radiotherapy versus standard of care palliative treatment in patients with oligometastatic cancers (SABRCOMET): a randomised, phase 2, open-label trial. Lancet 2019;393:2051-8.

22. Palma DA, Olson R, Harrow S, et al. Stereotactic Ablative Radiotherapy for the Comprehensive Treatment of Oligometastatic Cancers: Long-Term Results of the SABR-COMET Phase II Randomized Trial. J Clin Oncol 2020;38:2830-8.

23. Gomez DR, Blumenschein GR Jr, Lee JJ, et al. Local consolidative therapy versus maintenance therapy or observation for patients with oligometastatic non-smallcell lung cancer without progression after first-line systemic therapy: a multicentre, randomised, controlled, phase 2 study. Lancet Oncol 2016;17:1672-82.

24. Gomez DR, Tang C, Zhang J, et al. Local Consolidative Therapy Vs. Maintenance Therapy or Observation for Patients With Oligometastatic Non-Small-Cell Lung Cancer: Long-Term Results of a Multi-Institutional, Phase II, Randomized Study. J Clin Oncol 2019;37:1558-65.

25. Iyengar P, Wardak Z, Gerber DE, et al. Consolidative Radiotherapy for Limited Metastatic Non-Small-Cell Lung Cancer: A Phase 2 Randomized Clinical Trial. JAMA Oncol 2018;4:e173501.

26. Ost P, Reynders D, Decaestecker K, et al. Surveillance or Metastasis-Directed Therapy for Oligometastatic Prostate Cancer Recurrence: A Prospective, Randomized, Multicenter Phase II Trial. J Clin Oncol 2018;36:446-53.

27. Phillips R, Shi WY, Deek M, et al. Outcomes of Observation vs Stereotactic Ablative Radiation for
Oligometastatic Prostate Cancer: The ORIOLE Phase 2 Randomized Clinical Trial. JAMA Oncol 2020;6:650-9.

28. Ruers T, Van Coevorden F, Punt CJ, et al. Local Treatment of Unresectable Colorectal Liver Metastases: Results of a Randomized Phase II Trial. J Natl Cancer Inst 2017;109:djx015.

29. Wen PY, Loeffler JS. Management of brain metastases. Oncology (Williston Park). 1999;13:941-54, 57-61; discussion 61-2, 9.

30. Nayak L, Lee EQ, Wen PY. Epidemiology of brain metastases. Curr Oncol Rep 2012;14:48-54.

31. Scoccianti S, Ricardi U. Treatment of brain metastases: review of phase III randomized controlled trials. Radiother Oncol 2012;102:168-79.

32. Johnson JD, Young B. Demographics of brain metastasis. Neurosurg Clin N Am 1996;7:337-44.

33. Anzalone N, Essig M, Lee SK, et al. Optimizing contrastenhanced magnetic resonance imaging characterization of brain metastases: relevance to stereotactic radiosurgery. Neurosurgery 2013;72:691-701.

34. Stewart DJ. A critique of the role of the blood-brain barrier in the chemotherapy of human brain tumors. J Neurooncol 1994;20:121-39.

35. Gaspar L, Scott C, Rotman M, et al. Recursive partitioning analysis (RPA) of prognostic factors in three Radiation Therapy Oncology Group (RTOG) brain metastases trials. Int J Radiat Oncol Biol Phys 1997;37:745-51.

36. Sperduto PW, Berkey B, Gaspar LE, et al. A new prognostic index and comparison to three other indices for patients with brain metastases: an analysis of 1,960 patients in the RTOG database. Int J Radiat Oncol Biol Phys 2008;70:510-4.

37. Sperduto PW, Kased N, Roberge D, et al. Summary report on the graded prognostic assessment: an accurate and facile diagnosis-specific tool to estimate survival for patients with brain metastases. J Clin Oncol 2012;30:419-25.

38. Sperduto PW, Mesko S, Li J, et al. Survival in Patients With Brain Metastases: Summary Report on the Updated Diagnosis-Specific Graded Prognostic Assessment and Definition of the Eligibility Quotient. J Clin Oncol 2020;38:3773-84.

39. Barnholtz-Sloan JS, Yu C, Sloan AE, et al. A nomogram for individualized estimation of survival among patients with brain metastasis. Neuro Oncol 2012;14:910-8.

40. Patchell RA, Tibbs PA, Walsh JW, et al. A randomized trial of surgery in the treatment of single metastases to the brain. N Engl J Med 1990;322:494-500.

41. Noordijk EM, Vecht CJ, Haaxma-Reiche H, et al. The 
choice of treatment of single brain metastasis should be based on extracranial tumor activity and age. Int J Radiat Oncol Biol Phys 1994;29:711-7.

42. Patchell RA, Tibbs PA, Regine WF, et al. Postoperative radiotherapy in the treatment of single metastases to the brain: a randomized trial. JAMA 1998;280:1485-9.

43. Leksell L. Stereotactic radiosurgery. J Neurol Neurosurg Psychiatry 1983;46:797-803.

44. Lasak JM, Gorecki JP. The history of stereotactic radiosurgery and radiotherapy. Otolaryngol Clin North Am 2009;42:593-9.

45. Kondziolka D, Patel A, Lunsford LD, et al. Stereotactic radiosurgery plus whole brain radiotherapy versus radiotherapy alone for patients with multiple brain metastases. Int J Radiat Oncol Biol Phys 1999;45:427-34.

46. National Comprehensive Cancer Network. Central Nervous System Cancers (Version 3.2020) [Internet]. 2020 Sept 11 [cited 2021 Feb 2]. Available online: https://www. nccn.org/professionals/physician_gls/pdf/cns.pdf

47. Andrews DW, Scott CB, Sperduto PW, et al. Whole brain radiation therapy with or without stereotactic radiosurgery boost for patients with one to three brain metastases: phase III results of the RTOG 9508 randomised trial. Lancet 2004;363:1665-72.

48. Aoyama H, Shirato H, Tago M, et al. Stereotactic radiosurgery plus whole-brain radiation therapy vs stereotactic radiosurgery alone for treatment of brain metastases: a randomized controlled trial. JAMA 2006;295:2483-91.

49. Aoyama H, Tago M, Shirato H, et al. Stereotactic Radiosurgery With or Without Whole-Brain Radiotherapy for Brain Metastases: Secondary Analysis of the JROSG 99-1 Randomized Clinical Trial. JAMA Oncol 2015;1:457-64.

50. Chang EL, Wefel JS, Hess KR, et al. Neurocognition in patients with brain metastases treated with radiosurgery or radiosurgery plus whole-brain irradiation: a randomised controlled trial. Lancet Oncol 2009;10:1037-44.

51. Kocher M, Soffietti R, Abacioglu U, et al. Adjuvant wholebrain radiotherapy versus observation after radiosurgery or surgical resection of one to three cerebral metastases: results of the EORTC 22952-26001 study. J Clin Oncol 2011;29:134-41.

52. Soffietti R, Kocher M, Abacioglu UM, et al. A European Organisation for Research and Treatment of Cancer phase III trial of adjuvant whole-brain radiotherapy versus observation in patients with one to three brain metastases from solid tumors after surgical resection or radiosurgery: quality-of-life results. J Clin Oncol 2013;31:65-72.

53. Sahgal A, Aoyama H, Kocher M, et al. Phase 3 trials of stereotactic radiosurgery with or without whole-brain radiation therapy for 1 to 4 brain metastases: individual patient data meta-analysis. Int J Radiat Oncol Biol Phys 2015;91:710-7.

54. Brown PD, Jaeckle K, Ballman KV, et al. Effect of Radiosurgery Alone vs Radiosurgery With Whole Brain Radiation Therapy on Cognitive Function in Patients With 1 to 3 Brain Metastases: A Randomized Clinical Trial. JAMA 2016;316:401-9.

55. Hong AM, Fogarty GB, Dolven-Jacobsen K, et al. Adjuvant Whole-Brain Radiation Therapy Compared With Observation After Local Treatment of Melanoma Brain Metastases: A Multicenter, Randomized Phase III Trial. J Clin Oncol 2019;37:3132-41.

56. Rusthoven CG, Yamamoto M, Bernhardt D, et al. Evaluation of First-line Radiosurgery vs WholeBrain Radiotherapy for Small Cell Lung Cancer Brain Metastases: The FIRE-SCLC Cohort Study. JAMA Oncol 2020;6:1028-37.

57. Choosing Wisely. ASTRO releases second list of five radiation oncology treatments to question, as part of national Choosing Wisely campaign [Internet]. 2014 Sept 15 [cited 2021 Mar 8]. Available online: http://www. choosingwisely.org/astro-releases-second-list/

58. Karlsson B, Hanssens P, Wolff R, et al. Thirty years' experience with Gamma Knife surgery for metastases to the brain. J Neurosurg 2009;111:449-57.

59. Serizawa T, Yamamoto M, Sato Y, et al. Gamma Knife surgery as sole treatment for multiple brain metastases: 2-center retrospective review of 1508 cases meeting the inclusion criteria of the JLGK0901 multi-institutional prospective study. J Neurosurg 2010;113 Suppl:48-52.

60. Chang WS, Kim HY, Chang JW, et al. Analysis of radiosurgical results in patients with brain metastases according to the number of brain lesions: is stereotactic radiosurgery effective for multiple brain metastases? J Neurosurg 2010;113 Suppl:73-8.

61. Hughes RT, Masters AH, Mc'Tyre ER, et al. Initial SRS for Patients With 5 to 15 Brain Metastases: Results of a Multi-Institutional Experience. Int J Radiat Oncol Biol Phys 2019;104:1091-8.

62. Yamamoto M, Higuchi Y, Sato Y, et al. Stereotactic Radiosurgery for Patients with 10 or More Brain Metastases. Prog Neurol Surg 2019;34:110-24.

63. Yamamoto M, Serizawa T, Shuto T, et al. Stereotactic radiosurgery for patients with multiple brain metastases 
(JLGK0901): a multi-institutional prospective observational study. Lancet Oncol 2014;15:387-95.

64. Meyers CA, Brown PD. Role and relevance of neurocognitive assessment in clinical trials of patients with CNS tumors. J Clin Oncol 2006;24:1305-9.

65. Meyers CA, Wefel JS. The use of the mini-mental state examination to assess cognitive functioning in cancer trials: no ifs, ands, buts, or sensitivity. J Clin Oncol 2003;21:3557-8.

66. Li J, Ludmir EB, Wang Y, et al. Stereotactic Radiosurgery versus Whole-brain Radiation Therapy for Patients with 4-15 Brain Metastases: A Phase III Randomized Controlled Trial. Int J Radiat Oncol Biol Phys 2020;108:S21-2.

67. Ayala-Peacock DN, Peiffer AM, Lucas JT, et al. A nomogram for predicting distant brain failure in patients treated with gamma knife stereotactic radiosurgery without whole brain radiotherapy. Neuro Oncol 2014;16:1283-8.

68. Sawrie SM, Guthrie BL, Spencer SA, et al. Predictors of distant brain recurrence for patients with newly diagnosed brain metastases treated with stereotactic radiosurgery alone. Int J Radiat Oncol Biol Phys 2008;70:181-6.

69. Comparing Hippocampal-Avoidant Whole Brain Radiotherapy to Stereotactic Radiosurgery for Patients with 10 or Fewer Brain Metastases from Small Cell Lung Cancer (NRG-CC009) [Internet]. 2021 Mar 9 [cited 2021 Mar 11]. Available online: https://www.nrgoncology.org/ Home/News/Post/comparing-hippocampal-avoidantwhole-brain-radiotherapy-to-stereotactic-radiosurgeryfor-patients-with-10-or-fewer-brain-metastases-fromsmall-cell-lung-cancer-nrg-cc009

70. Shaw E, Scott C, Souhami L, et al. Single dose radiosurgical treatment of recurrent previously irradiated primary brain tumors and brain metastases: final report of RTOG protocol 90-05. Int J Radiat Oncol Biol Phys 2000;47:291-8.

71. Vogelbaum MA, Angelov L, Lee SY, et al. Local control of brain metastases by stereotactic radiosurgery in relation to dose to the tumor margin. J Neurosurg 2006;104:907-12.

72. Wiggenraad R, Verbeek-de Kanter A, Kal HB, et al. Dose-effect relation in stereotactic radiotherapy for brain metastases. A systematic review. Radiother Oncol 2011;98:292-7.

73. Marcrom SR, McDonald AM, Thompson JW, et al. Fractionated stereotactic radiation therapy for intact brain metastases. Adv Radiat Oncol 2017;2:564-71.

74. Minniti G, D'Angelillo RM, Scaringi C, et al. Fractionated stereotactic radiosurgery for patients with brain metastases. J Neurooncol 2014;117:295-301.
75. Baschnagel AM, Meyer KD, Chen PY, et al. Tumor volume as a predictor of survival and local control in patients with brain metastases treated with Gamma Knife surgery. J Neurosurg 2013;119:1139-44.

76. Kim IK, Starke RM, McRae DA, et al. Cumulative volumetric analysis as a key criterion for the treatment of brain metastases. J Clin Neurosci 2017;39:142-6.

77. Hirshman BR, Wilson B, Ali MA, et al. Superior Prognostic Value of Cumulative Intracranial Tumor Volume Relative to Largest Intracranial Tumor Volume for Stereotactic Radiosurgery-Treated Brain Metastasis Patients. Neurosurgery 2018;82:473-80.

78. Joshi RS, Hirshman BR, Ali MA, et al. Prognostic Importance of Cumulative Intracranial Tumor Volume in Patients with Gastrointestinal Brain Metastasis Treated with Stereotactic Radiosurgery. World Neurosurg 2019;121:e747-54.

79. Mok TS, Wu YL, Ahn MJ, et al. Osimertinib or PlatinumPemetrexed in EGFR T790M-Positive Lung Cancer. N Engl J Med 2017;376:629-40.

80. Reungwetwattana T, Nakagawa K, Cho BC, et al. CNS Response to Osimertinib Versus Standard Epidermal Growth Factor Receptor Tyrosine Kinase Inhibitors in Patients With Untreated EGFR-Mutated Advanced NonSmall-Cell Lung Cancer. J Clin Oncol 2018. [Epub ahead of print]. doi: 10.1200/JCO.2018.78.3118.

81. Peters S, Camidge DR, Shaw AT, et al. Alectinib versus Crizotinib in Untreated ALK-Positive Non-Small-Cell Lung Cancer. N Engl J Med 2017;377:829-38.

82. Camidge DR, Kim DW, Tiseo M, et al. Exploratory Analysis of Brigatinib Activity in Patients With Anaplastic Lymphoma Kinase-Positive Non-Small-Cell Lung Cancer and Brain Metastases in Two Clinical Trials. J Clin Oncol 2018;36:2693-701.

83. Lin NU, Carey LA, Liu MC, et al. Phase II trial of lapatinib for brain metastases in patients with human epidermal growth factor receptor 2-positive breast cancer. J Clin Oncol 2008;26:1993-9.

84. Lin NU, Dieras V, Paul D, et al. Multicenter phase II study of lapatinib in patients with brain metastases from HER2-positive breast cancer. Clin Cancer Res 2009;15:1452-9.

85. Bachelot T, Romieu G, Campone M, et al. Lapatinib plus capecitabine in patients with previously untreated brain metastases from HER2-positive metastatic breast cancer (LANDSCAPE): a single-group phase 2 study. Lancet Oncol 2013;14:64-71.

86. Freedman RA, Gelman RS, Anders CK, et al. TBCRC 
022: A Phase II Trial of Neratinib and Capecitabine for

Patients With Human Epidermal Growth Factor Receptor 2-Positive Breast Cancer and Brain Metastases. J Clin Oncol 2019;37:1081-9.

87. Murthy RK, Loi S, Okines A, et al. Tucatinib, Trastuzumab, and Capecitabine for HER2-Positive Metastatic Breast Cancer. N Engl J Med 2020;382:597-609.

88. Lin NU, Borges V, Anders C, et al. Intracranial Efficacy and Survival With Tucatinib Plus Trastuzumab and Capecitabine for Previously Treated HER2-Positive Breast Cancer With Brain Metastases in the HER2CLIMB Trial. J Clin Oncol 2020;38:2610-9.

89. McArthur GA, Maio M, Arance A, et al. Vemurafenib in metastatic melanoma patients with brain metastases: an open-label, single-arm, phase 2, multicentre study. Ann Oncol 2017;28:634-41.

90. Davies MA, Saiag P, Robert C, et al. Dabrafenib plus trametinib in patients with BRAF(V600)-mutant melanoma brain metastases (COMBI-MB): a multicentre, multicohort, open-label, phase 2 trial. Lancet Oncol 2017;18:863-73

91. Brastianos PK, Twohy E, Anders CK, et al. Alliance A071701: Genomically guided treatment trial in brain

Cite this article as: Chaung $\mathrm{KV}$, Sloan $\mathrm{AE}$, Choi S. Limited brain metastases: a narrative review. Ann Palliat Med 2021;10(5):6016-6027. doi: 10.21037/apm-21-363 metastases. J Clin Oncol 2020;38:TPS2573.

92. Goldberg SB, Gettinger SN, Mahajan A, et al.

Pembrolizumab for patients with melanoma or non-smallcell lung cancer and untreated brain metastases: early analysis of a non-randomised, open-label, phase 2 trial. Lancet Oncol 2016;17:976-83.

93. Tawbi HA, Forsyth PA, Algazi A, et al. Combined Nivolumab and Ipilimumab in Melanoma Metastatic to the Brain. N Engl J Med 2018;379:722-30.

94. Rishi A, Yu HM. Current Treatment of Melanoma Brain Metastasis. Curr Treat Options Oncol 2020;21:45.

95. Reynders K, Illidge T, Siva S, et al. The abscopal effect of local radiotherapy: using immunotherapy to make a rare event clinically relevant. Cancer Treat Rev 2015;41:503-10.

96. Acharya S, Mahmood M, Mullen D, et al. Distant intracranial failure in melanoma brain metastases treated with stereotactic radiosurgery in the era of immunotherapy and targeted agents. Adv Radiat Oncol 2017;2:572-80.

97. Lehrer EJ, Peterson J, Brown PD, et al. Treatment of brain metastases with stereotactic radiosurgery and immune checkpoint inhibitors: An international meta-analysis of individual patient data. Radiother Oncol 2019;130:104-12. 